\title{
Editorial note to: \\ H. P. Robertson, Relativistic cosmology
}

\author{
George Ellis
}

Published online: 29 June 2012

(C) Springer Science+Business Media, LLC 2012

Keyword Relativistic cosmology - Line element - Robertson-Walker metric · Stationary and non-stationary universes $\cdot$ Golden Oldie

\section{The context}

H. P. Robertson's paper "Relativistic Cosmology" [48] was the first review paper on the subject of relativistic cosmological models:

"This report deals with the attempts which have been made during the past decade and a half to solve the general problem of the structure of the universe as a whole."

Such models had been initiated by Einstein's 1917 paper [12] on the Einstein static solution, ${ }^{1}$ and then developed by a small group of pioneers: Einstein, de Sitter, Friedmann, Lanczos, Weyl, Lemaître, Robertson, Tolman, and Eddington. A second wave of pioneers-von Laue, Heckmann, McCrea, McVittie, Whittaker, Ward, Milne, and Walker $^{2}$ - joined in after the idea of an expanding universe became accepted in 1931, providing a sound theoretical basis for Hubble's observations of the magnitude-redshift relation for galaxies. Robertson's article was written 16 years after the first General Relativistic cosmological model and 11 years after the first expanding universe models

\footnotetext{
1 All the early papers mentioned here are referenced in Robertson's review article [48].

2 Milne and Walker made their significant contributions to the foundations of cosmology after Robertson's review article was completed, so are not referenced there.
}

The republication of the original paper can be found in this issue following the editorial note and online via doi:10.1007/s10714-012-1401-0.

G. Ellis $(\bowtie)$

Mathematics Department, University of Cape Town, Rondebosch, Cape Town 7700, South Africa e-mail: George.Ellis@uct.ac.za 
$[25,26]$, but it appeared only two years after the idea of an expanding universe gained widespread acceptance, largely because of Eddington's republication of Lemaitre's 1927 paper [36]. ${ }^{3}$ The publication of this article in Reviews of Modern Physics signals recognition of relativistic cosmology as a legitimate scientific theory.

The paper is a clearly written statement of how things were seen at the time, and raises many of the issues that are main items of concern today. However some aspects of the way it is presented are different than the way they would be discussed today, and some major present day themes are missing. There are of course now numerous excellent survey articles and books discussing observational, physical, and astrophysical aspects of cosmology, but not nearly so many discussing cosmology from a relativistic viewpoint. Some that stand out as doing so are Bondi's book [7], Ehlers' survey article [10], the Varenna lectures [13,23], Rindler's book [46], the book by Peters and Uzan [44], and the recent text [20].

\section{The family of models}

Cosmological models are based in general relativity theory, so Robertson starts off by summarizing this theory: through the field equations (1.2) (including a cosmological constant $\lambda$ ), the metric tensor (1.1) is contingent on the physical content of spacetime, represented as a perfect fluid (1.7). He claims this represents a precise formulation of Mach's principle, but that is no longer agreed: Mach's principle remains a contested item today [2]. He justifies spatial homogeneity of a background model on the basis of observations of 'extragalactic nebulae' (i.e galaxies); this model is an ideal on which are to be superposed local irregularities representing the actual distribution of matter and energy in the actual world. There is supposed to exist a well defined mean motion of matter in each region of cosmic spacetime: that is, the universe is a coherent whole (in modern jargon: it is not chaotic). He introduces comoving coordinates and a cosmic time $t$ defining simultaneity. On this basis he introduces the standard spatially homogeneous and isotropic metric (2.2), (2.3) for arbitrary spatial curvature $k$, but preferring models with $k=+1$.

The field equations applied to this metric give the equations (3.2) determining the total energy density and pressure of matter and radiation from time derivatives of the scale factor $R(t)$. He does not point out that only two of the three equations (3.2), (3.3) are independent, nor does he eliminate the quantity $R^{\prime 2}$ between the equations (3.2) to give the Raychaudhuri equation determining $R^{\prime \prime}$ from the active gravitational mass density $\rho+3 p / c^{2}$ :

$$
6 R^{\prime \prime} / R c^{2}=2 \lambda-\kappa\left(\rho c^{2}+3 p\right)
$$

This is the equation that is crucial in singularity theorems for these models (see $[10,13])$.

Robertson comments that the matter contained in a comoving volume can never cross the boundary, and the rate radiation enters such a volume is balanced by that

$\overline{3 \text { See }[5,15,16,39] \text { for this history. }}$ 
leaving. He defines a total mass $M$ and energy $E$ (3.5) for such a volume, the latter generally not being constant because it is composed of both matter and radiation [see (9.1)]; rather it satisfies the thermodynamic law (3.6), which follows from the conservation equation (3.3). Neither $M$ nor $E$ is constant if matter is transformed into radiation [see (9.9)].

At this point no equations of state have been given for the matter, so Robertson suggests running the field equations backwards to determine the density $\rho$ and pressure $p$ from arbitrary $k, \lambda$, and $R(t)$ but subject to the inequality $p \geq 0$. He then quickly retreats from this proposal, ${ }^{4}$ which is currently highly disfavored because it produces non-physical solutions of the field equations, and proposes integration of the Friedmann equation [the first of (3.2)] to give $t(R)$ by the quadrature $(3.7,3.8)$, with $E$ given by (3.5). Before turning to solutions, he shows how to integrate timelike and null geodesics, the latter giving a velocity-distance relation (4.6) leading to a linear redshift-distance relation (4.9) given in terms of spatial distance $l$ and the wavelength change $\Delta \lambda / \lambda .{ }^{5}$ He then identifies the existence of observational limits, characterized by the radius $l(t)$ of the observable universe at time $t .{ }^{6}$ He comments on being able to see round the universe if $k=+1$ and one has the elliptical identification, and introduces the idea of luminosity distance in (4.12). Thus the basics of cosmological magnituderedshift observations are all there; but no expressions are given for number counts. ${ }^{7}$

\section{Solutions}

Turning to solutions of these equations, Robertson derives the Einstein static solution for matter with arbitrary pressure. It obeys

$$
2 \lambda=\kappa\left(\rho_{0} c^{2}+3 p_{0}\right), \quad 2 k / R_{0}^{2}=\kappa\left(\rho_{0} c^{2}+p_{0}\right),
$$

showing that necessarily $\lambda>0, k=+1$, if

$$
\begin{aligned}
& \rho_{\text {grav }}:=\left(\rho_{0} c^{2}+3 p_{0}\right) \geq 0, \\
& \rho_{\text {inert }}:=\left(\rho_{0} c^{2}+p_{0}\right) \geq 0
\end{aligned}
$$

respectively. He calculates its radius from Hubble's value for the density of matter ${ }^{8}$ and determines the time for light to circumnavigate it, and shows how to represent it

\footnotetext{
4 Nowadays called the Synge trick or Synge's g-method.

5 The paper preceded the definition of redshift $z$ in current use today. His expression only reduces to zero as $l \rightarrow 0$ if $v_{0}=0$ or $v_{0}=2 c$; also the second order term in (4.9) is not the observationally determinable deceleration parameter, as suggested by his comment at the top of page 69 . These issues were sorted out later by McVittie, McCrea, and Heckmann.

${ }^{6} \mathrm{He}$ is in effect defining the particle horizon, which was the subject of much confusion until its nature was cleared up by Rindler in 1956 [45]. Similarly, in the paragraph after (6.4), Robertson refers to the event horizon in the de Sitter spacetime, without giving it any name. The name, and explanation, were given later in the same paper by Rindler.

7 See Sandage [51] for a derivation of all these results.

8 Somewhat higher than present estimates.
} 
as a cylinder imbedded in 5-dimensional flat spacetime. ${ }^{9}$ But this is not a good model universe as it has no redshifts and is unstable.

He then presents the expanding de Sitter solution in the stationary $(k=0)$ frame (6.1), showing it must be an empty spacetime if we apply the energy conditions $\rho \geq 0$, $p \geq 0$. He shows how to represent it as part of a hyperboloid imbedded in 5-dimensional flat spacetime, and considers observational horizons in this model. He gives the $k=+1$ expanding form (6.6) and $k=-1$ expanding form (6.7), ${ }^{10}$ but does not show which parts of the hyperboloid they cover. He does not give the static form originally found by de Sitter (which is not of form (2.2)), ${ }^{11}$ but does comment that one has to use Weyl's postulate to get the expanding form, the problem being that it is a spacetime of constant curvature and consequently has no intrinsically preferred timelike vector fields. Finally he gives the flat-space linearly expanding empty solution that we now identify as Milne's universe, characterized as the only other empty universe [i.e. the only other vacuum solution with metric (2.2)].

He then turns to an extensive investigation of expanding, non-empty solutions. The Friedmann equation can be treated as an energy equation with an effective potential that depends on the quantities $k$ and $\lambda$ (see Figs. 3 and 4); the only points where $R^{\prime}$ can change sign are where $D=0$. Consequently one gets solutions of various types as laid out in Tables 1 and 2 of Robertson's paper, where the universe types are labeled $M=$ monotonic, $O=$ oscillating, and $A=$ asymptotic, as follows:

\begin{tabular}{|c|c|c|c|}
\hline & Initial state & Continuation & Final state \\
\hline$M_{1}$ & Singular & expands & Infinity \\
\hline$M_{2}$ & Infinity & $\begin{array}{c}\text { decreases to minimum, } \\
\text { re-expands }\end{array}$ & Infinity \\
\hline$O$ & Singular & $\begin{array}{c}\text { expands to maximum, } \\
\text { re-collapses }\end{array}$ & Singular \\
\hline$A_{1}$ & Singular & expands & Einstein static \\
\hline$A_{2}$ & Einstein static & expands & Infinity \\
\hline$E$ & Einstein static & static & Einstein static \\
\hline
\end{tabular}

It is clear that type $M_{2}$, called 'monotonic of the second kind', is in fact not monotonic at all! (see the sentence after (7.6)).

- Section 7 looks at models with $p \geq 0$, firstly with $k=+1$ for various values of $\lambda$ and particularly for models with $d p / d R \leq 0$ (pressure does not increase as the universe expands), then for models with $k=0$ and $k=-1$, as set out in Table 1;

- Section 8 looks at models with "energy conserved", which is code for pressure-free matter and no radiation $[E=$ const implies $p=u=0$ by (3.6) and (9.1)], as set out in Table 2; particular cases are Lemaitre's case of type $A_{1}$, Einstein's case of type $O$, and the Einstein-de Sitter case of type $M_{1}$ with $k=0, \lambda=0$;

\footnotetext{
9 This plays an important role in Penrose's later conformal representations of Robertson-Walker cosmologies.

10 The singular form of the de Sitter universe is the Lanczos form (6.7); there is a misprint here just above (7.5), where Robertson's paper says (6.6).

11 The relation of these various coordinates is carefully explained by Schrödinger in [50].
} 
- Section 9 has two parts. ${ }^{12}$ The first part looks at pressure free matter with non-zero radiation ( $M$ is constant but $E$ is not constant), including Lemaitre's ever-expanding non-singular universe with $\lambda>0, k=+1$; at pure radiation models (i.e. $M=0$ ), including an analogue of the Lemaitre eternally expanding universe; and at matter with non-zero pressure $p_{m}$ due to random motions of galaxies.

- Section 9, second part looks at pressure-free matter being continually transformed into radiation by annihilation of matter, leading to a phenomenological law (9.9) for the change of radiation density $u$ and energy $E$ with scale factor $R$. These models are special cases of those considered in Section 7.

- Section 10 looks at the Condensation problem: could a fluctuation of some kind lead an initially Einstein static universe to start expanding? Power series expansions show that a diminution of pressure or increase in proper mass or density of matter would make such a universe start expanding, leading to a realization of what is now called an emergent universe [19,22].

Two comments on the latter are in order. Firstly, much simpler arguments would have been possible if Robertson had derived the Raychaudhuri equation (1); these results follow immediately from that equation together with the conservation equation (3.3). Secondly, he has not considered combining this discussion with the previous section: that is, asking what the effect would be of pressure-free matter starting at some time $t_{i}$ to annihilate to give radiation in an Einstein static universe. Then $\rho$ will remain constant while $p$ will change; hence the first of (3.2) will still balance but the second will not. From Eq. (1), initially

$$
\kappa\left(\rho_{0} c^{2}+3 p_{0}\right)=2 \lambda .
$$

Substituting in Eq. (2), at any later time

$$
6 R^{\prime \prime} / R c^{2}=3 \kappa\left(p_{0}-p\right)+\kappa\left(\rho_{0}-\rho\right) c^{2},
$$

so if annihilation of matter takes place from a zero pressure initial state $\left(p_{0}=0\right)$ with conservation of energy (3.4): $\rho_{0}=\rho$, then

$$
6 R^{\prime \prime} / R c^{2}=-3 \kappa p
$$

and the universe will start to collapse because $p_{r}>0$ (by (1.8)). Hence this works in the opposite of the intended direction.

The last paragraph of Section 10 has a distinctly modern flavor: Lemaitre had considered models of structure formation in an ever expanding universe, where matter at a large distance will recede from a growing condensation, but nearer matter will fall back in. This parallels later investigations by Rees and Silk. Also in Section 9 (just above Table III) Robertson comments on the possibility of dark matter: a highly topical issue. In Section 10, he comments on the timescale problem, which was acute

\footnotetext{
12 The heading of this section is very confusing, by conflating the content of two subsections: matter is not conserved if there is annihilation of matter.
} 
at the time because Hubble's value (4.10) for the Hubble parameter was too low. This is one of the reasons Robertson prefers models with a positive cosmological constant.

\section{Appendices}

Appendix A gives connection and curvature tensor components for the line elements (2.3), (2.3); Appendix B summarizes properties of spaces of constant curvature, and gives the coordinate form (B.4) for their metric tensor. Appendix C summarizes the properties of symmetry groups, giving Killing's equations (C.4) but not actually solving them except for the case of stationary models. He also gives conditions for a universe to be periodic. Appendix $\mathbf{D}$ gives helpful imbedding diagrams of $k=+1$ models in 5-dimensional flat spacetimes (Figure 7), with special cases having been already presented (Figures 1 and 2). This is a very useful feature of the paper, not often presented in more recent texts. Appendix E summarizes Tolman's relativistic thermodynamics, giving a covariant form of the second law of thermodynamics, and considering reversible and irreversible processes in cosmology.

Finally there is a Bibliography with brief notes, a very useful listing of the early papers in cosmology. Two cautionary notes: there are some extra works referred to in footnotes that are not listed here, for example Eddington's paper in footnote 24, Zwicky's in footnote 54, and Milne's paper in footnote 55. Secondly, Robertson did indeed include the expanding universe forms for all $k$ in his paper in 1929 [47], as stated here, but he did not at that time realize their significance: like all the other major protagonists except Friedmann and Lemaitre, he was looking for stationary models $[15,16]$. However the 1929 paper is significant in that he showed there that all these models are both conformally flat and imbeddable in 5-dimensional flat spacetime.

\section{Later developments}

While the basics have remained unchanged, huge developments have taken place in observations and testing and in physical understanding of cosmological models. They have become a part of mainstream physics. Some key later developments are as follows.

Geometry Developing from Milne's kinematic relativity, Robertson [49] and Walker [58] showed that the standard metric of cosmology $(2.2,2.3)$ can be derived from purely kinematic assumptions: the derivation does not depend on the field equations. These papers led to these metrics being commonly designated Robertson-Walker (RW) metrics. The papers set up the group theory structure to put the symmetry properties of these metrics on a solid footing but did not actually derive the Killing vectors or group structure constants. ${ }^{13}$ Walker also showed the fundamental result that isotropy everywhere implies spatial homogeneity [57,59]. Later Ehlers gave an elegant derivation of this result [10].

\footnotetext{
13 Much later it was shown [31,21] that the $k=-1$ models admitted Bianchi groups of types types V and $V I I_{h}$. This was surprising as it was commonly assumed their symmetry group would be Bianchi type $V I I I$, because of its close relation to Bianchi $I X$, which was well known to be the symmetry group of the $k=+1$ models.
} 
For a while it was common to suppose spatial homogeneity was explained by an assumed Cosmological Principle [7]: the explicit a priori assumption that the universe must be spatially homogeneous and isotropic. Sometimes this was weakened to a Copernican principle: the assumption that we must live in a typical place in the universe. Together with the observed isotropy of observations about us, this then led (by Walker's theorem [57,59]) to the deduction we must live in a RW spacetime. Recent work has changed the previously untestable Copernican assumption [17] into an observationally testable hypothesis; this important development is discussed comprehensively in [41].

Topology The 'natural' topology for the spatial sections is the simply connected one. However there are other topologies possible for each spatial curvature [14]. Robertson considered the elliptic topology of $k=+1$ spaces; since then, all the topologies for the cases $k=0$ and $k=+1$ have been found, and catalogues created of the infinite number of possibilities in the case $k=-1$. Any of them are theoretically possible: there is no known principle that will determine which of them should occur in the real universe. Various observational tests can in principle detect which may be the case $[35,40]$.

Kinematics Robertson emphasizes the Weyl postulate [60] as the basis of kinematics in these models. In the paragraph above (2.1) Robertson says

"the space $t=$ const, the coordinates of which... shall be orthogonal to this (normal) congruence of geodesics. The possibility of thus introducing in a natural and significant way this cosmic time $t$ we consider as guaranteed by Weyl's postulate..."

In fact, this statement is incorrect. To draw this conclusion, which leads to the line element (2.1), one must add one more assumption: that Weyl's pencil of geodesics has zero rotation. When rotation is nonzero, spaces orthogonal to the family of geodesics in question do not exist [10]. Rotation was first recognised as a possible ingredient of motion of cosmic matter by Gödel [28,29], and an invariant definition of rotation for a congruence of timelike curves was given only in 1961 by Ehlers [10]. A generalisation of the R-W models to an exact expanding solution with a perfect fluid source and nonzero rotation remains unknown until today, in consequence of prohibitive computational difficulties (if shear-free such solutions existed, such models would be easy to find; but there are none [29]).

In any case, the Weyl postulate is only necessary in maximally symmetric spacetimes (Minkowski, de Sitter, and anti-de Sitter); in any realistic cosmology, the energy inequality (4) will be satisfied and hence a unique congruence of fundamental world lines will be defined by having as tangent vector $u^{a}$ the normalized future-directed timelike eigenvector of the Ricci tensor. The RW spacetimes can then be characterized as precisely the perfect fluid spacetimes for which the acceleration $\dot{u}^{a}$, shear $\sigma_{a b}$, and vorticity $\omega^{a}$ of this vector field is zero [10,13]. Robertson in his 1929 paper [47] showed they are conformally flat, but an inverse result can be proved: in the case of perfect fluids with either zero pressure $(p=0)$ or a non-degenerate barotropic 
equation of state $(p=p(\rho), d p / d \rho \neq 0$ ) one can also characterize them as precisely those spacetimes that are conformally flat $\left(C_{a b c d}=0\right)[13]$.

Matter and energy conditions Robertson considered a mixture of a perfect fluid plus radiation, with the following energy inequalities imposed at various stages:

$$
p \geq 0, \rho>0, d p / d R \leq 0
$$

By the conservation equation (3.3), when $d p / d \rho \geq 0$, the latter is equivalent to inequality (4). Indeed it has now become clear that rather than (8), the really significant energy inequalities are positive gravitational mass density (3) and positive inertial mass density (4); these are satisfied by all ordinary matter.

The crucial new feature then is the realization that a scalar field $\phi$ with potential $V(\phi)$ has an energy momentum tensor that satisfies (4) but can violate (3). This underlies the proposal of an inflationary era in the early universe.

Physics Inflation is a result of application of new physical ideas in the very early universe. Indeed this is one of the major changes since this review paper was written: successively new parts of physics have been brought into the cosmological story, as follows:

Table 1 Item 1 is discussed thoroughly by Robertson. It would not have been possible for cosmologists at that time to have investigated Items 3-5, as the relevant physics was not known then. However, with the benefit of hindsight, Item 2 was the great lost opportunity (how easy it is to say it in retrospect!). Robertson's paper explicitly includes radiation as well as matter, and even gives the dynamic equation governing radiation only universes [equation (9.6)], which sets the key timescale for expansion in the Hot Big Bang era (when $\lambda$ and $k$ can be ignored). Tolman discussed thermodynamics extensively, but did not see that ionization would have had to have occurred in the very early universe leading to matter-radiation equilibrium early on, and ending at decoupling of matter and radiation at the last scattering surface. Physicists could already at the time have predicted the existence of the cosmic black body background radiation and the early hot big bang era, but that had to wait until Gamow saw the connection in 1946 [1,27].

Table 1 Introduction of new physical domains into cosmology over time

\begin{tabular}{llll}
\hline & Physics & Application & Implication \\
\hline 1 & Gravitation & Dynamics of universe & Expansion history of universe \\
2 & Atomic physics & Ionization of universe, & Last scattering surface, \\
& Thermodynamics & Cosmic background radiation & Hot Big Bang era \\
3 & Nuclear physics & Nuclear reactions & Nucleosynthesis \\
4 & Particle physics and & Particle creation/annihilation, & Baryosynthesis, \\
& Quantum field theory & Scalar fields & Inflation \\
5 & Quantum gravity & Wave function of universe & Start of universe?
\end{tabular}


Dynamics Apart from the radiation dominated Hot Big Bang era, the inflationary era in the early universe where

$$
R^{\prime \prime}>0 \Leftrightarrow \rho_{\text {grav }}>0
$$

is the major new dynamical feature to be found since Robertson's paper. There are two technical improvements since then. Firstly, as already mentioned, derivation of the Raychaudhuri equation (1) made clear the dynamical significance of the active gravitational mass density $\rho+3 p / c^{2}$ and enabled simple proofs of singularity theorems for these models $[10,13]$. Secondly the array of solutions discussed here can be very nicely represented by various phase planes (see e.g. [46,20])); possibly the most illuminating are those for matter, radiation, and a cosmological constant by Ehlers and Rindler [11]. Finally also many anisotropic and inhomogeneous models have now been modeled, as well as those based on the RW geometry; they are summarized in $[34,43,20]$. They particularly include the Bianchi spatially homogeneous models [21], and the LTB spherically symmetric inhomogeneous models $[37,56,6]$. The latter is an exact solution that describes a spherically symmetric condensation or void with arbitrary profiles of mass density and of expansion velocity, and has been useful in cosmological studies. Papers based on this model were published in 1934 by Tolman [56] and Sen [53] that predicted that voids and other structures would have to form in an R-W background. Both these authors proved, using the LTB model, that a zeropressure $\mathrm{R}-\mathrm{W}$ model is unstable against the formation of voids and condensations; see Ref. [34] for more on this. Such voids were detected in 1978 [30,32] and confirmed in 1981 [33]. Equally promising is the Szekeres model [54,55,43] that has no symmetry in general and contains LTB as a subcase.

Inhomogeneities and structure formation A further major development has been linearized perturbation calculations, underlying studies of structure formation. These were initiated by Lifshitz in 1946 [38], but were plagued by gauge problems. A big step forward was Bardeen's gauge-covariant perturbation theory [3,42] which is widely used in studying growth of matter and radiation perturbations. ${ }^{14}$ This led to the discovery of acoustic peaks in the CBR angular spectrum and associated Baryon Acoustic Oscillation (BAO) peaks in the matter power spectrum, both of which have been observed.

Perturbation theories also underlie modeling of gravitational lenses, which have become very effective probes of inhomogeneity in the universe, and in particular the distribution of dark matter [52,4].

Observations The basics of redshift and luminosity distance were already given by Robertson; their relation to practical observations and number counts was discussed by Sandage [51]. The relation to angular diameter distances is given through Etherington's reciprocity theorem [24], which shows that specific intensity is independent of area distance [13], and hence underlies studies of CBR anisotropies [44,20]. The

$\overline{14}$ A $1+3$ covariant and gauge invariant formalism is also possible $[18,20]$. 
basics of horizons were already given by Robertson; their nature was very nicely clarified by Rindler [45].

The huge change since Robertson's paper has been progress in telescope and detector technology, accompanied by developments in computing facilities that can handle the vast amounts of data generated. This has resulted in the accumulation of detailed observational data in all wavelength bands for galaxies, radio sources, X-ray sources, and background radiation, as well as gravitational lensing studies of the distribution of matter $[44,20]$. This has refined our estimates of the Hubble constant and the density of matter in the universe, measured the CBR spectrum and anisotropy pattern over the sky, identified the CBR acoustic peaks and the BAO acoustic oscillations, and confirmed the presence of both dark matter and dark energy as major dynamical features of the recent universe. ${ }^{15}$

The consensus model - a perturbed RW geometry, with the background dynamics described by the Friedmann-Lemaître equations (3.2) presented in Robertson's paper-is now well supported by numerous observations that fit together in a coherent way.

The major themes Two of the major unsolved themes of today's cosmology were already present in Robertson's paper: namely the possible existence of dark matter, now regarded as well established, and the possible existence of an effective dark energy, also now observationally established. Much effort is being expended on trying to establish the nature of the dark matter, and to determine whether the dark energy is a cosmological constant or something more complex ("quintessence"). Robertson assumed it was preferred that $\lambda$ should be non zero, as indicated by present observations.

Robertson's considerations in the paragraph following (10.2) are worth quoting here. He considers there an $\mathrm{R}-\mathrm{W}$ model with $\lambda$ that was initially in a static phase described by the Einstein model, and was knocked out of equilibrium by fluctuations in density and pressure. He cites a result by Lemaître that the Universe would then need $10^{10}-10^{11}$ years to attain its present state. Then he says

"Such a time scale seems far too short for modern astrophysics, according to which the ages of the stars... may be of the order of $10^{12}$ years or more."

This comment is important in illustrating how astrophysical and cosmological understanding influence each other. This erroneous estimate of the ages of stars was replaced by more realistic ones once the mechanism by which stars emit energy was explained by Bethe in 1938; together with revised values of the Hubble constant, the age conflict was then resolved.

The basic model described by Robertson - an expanding universe model with a RW geometry and containing matter, radiation, and a cosmological constant—remains an excellent model of the universe at recent times, and indeed back to decoupling; but the present day understanding of times before decoupling is rather different, involving

\footnotetext{
15 The latter assumes the universe is indeed well described by a perturbed RW geometry. An LTB geometry could in principle do away with the need for dark energy [8,9]; this is one reason why tests of the Copernican principle [41] are important.
} 
much more complex physics. The model has had much detail filled in both as regards its matter content and the superimposed inhomogeneity structure. We have as it were dressed versions of the basic models presented in this excellent early survey paper, written by one of the pioneers of the field.

Acknowledgments I thank Andrzej Krasiński for helpful comments on previous drafts of this note.

\section{Howard Percy Robertson: a brief biography}

By Andrzej Krasiński, compiled and abstracted from Refs. [61] and [62].

Howard Percy Robertson was born on 27 January 1903 in Hoquiam, Washington, USA. He was known to his friends and colleagues as Bob. His parents were George Duncan Robertson and Anna McLeod, Americans of Scottish descent. George Robertson's family came from Maryland and he was an engineer who built bridges in the Washington area. Anna had attended Johns Hopkins and then become a nurse. H.P. was the eldest of his parents' five children, and when his father died in 1918 he had to help support the family.

H. P. Robertson entered the University of Washington in 1918. He had to earn money during his undergraduate years, so he was unable to participate in the usual student life. Originally he began an engineering course, but later he changed to major in pure mathematics. He was awarded a bachelor's degree in 1922 and a master's degree in 1923. Robertson's studies in mathematics and physics progressed under the influence of the mathematician E. T. Bell. His relation with Bell was stormy, but deep. It was Bell who urged him to enter graduate work at the California Institute of Technology.

Robertson got his doctorate from the California Institute of Technology in 1925 after submitting the dissertation "On the Dynamical Space-Time which Contains a Conformal Euclidean 3-Space”. His thesis advisor had been Harry Bateman. He was awarded a National Research Council Fellowship in mathematics to study in Germany where he spent the years 1925-1927. Most of that time he spent in Göttingen, but six months of it in Munich. He met Hilbert, Courant, Martin Schwarzschild, von Neumann, Wigner, Schrödinger, Heisenberg and Einstein. He impressed Courant and Hilbert with his mathematical skills, and, in addition to that, left an amusing mark of a rather exceptional type: " ... he rolled a barrel of beer through cobbled Munich streets at 2:00 A.M. and thus earned a police citation for 'disturbing the citizenry" " [62].

He returned to the California Institute of Technology in 1927, where he was appointed assistant professor of mathematics. Two years later he was appointed assistant professor at Princeton, where he worked for 18 years, later as associate professor and then full professor, in both the mathematics department and the physics department.

A second course of his career began in 1939 when World War II started. Although the United States was initially not involved in the war, Robertson became, under the urging of Richard Tolman, a member of a group which was to become part of the National Defense Research Committee. They studied the effectiveness of explosives and the mathematical theory of explosion damage. After the fall of France in May 1940, Robertson was given the position of liaison scientist with Britain. Over the next 
years he spent time in both Britain and the United States, then in 1943 he moved to Britain on a semi-permanent basis. In 1944 he was put in charge of a group which was assigned the task of studying German scientific technology. He remained in Europe until the middle of 1946 when he returned to Princeton. He was awarded the Medal of Merit in 1946 for "solving complex technical problems in the fields of bomb ballistics, penetrations and patterns, and enemy secret weapons" [62]. Subsequently, he was Director of the Weapons System Evaluation Group from 1950 to 1952, while continuing to teach relativity at Caltech, then from 1954 to 1956 he was back in Europe as Scientific Advisor to the NATO Commander. After returning to Caltech he was Chairman of the Defense Science Board and member of the President's Scientific Advisory Committee.

Among these activities he managed to make contributions to differential geometry, quantum theory, general relativity, and cosmology: “... he was interested in the foundations of physical theories, differential geometry, the theory of continuous groups, and group representations. He was particularly interested in the application of the latter three subjects to physical problems." [63].

His contributions to differential geometry came in papers such as: "The absolute differential calculus of a non-Pythagorean non-Riemannian space" (1924, [64]); "Transformations of Einstein spaces" (1925, [65]); "Dynamical space-times which contain a conformal Euclidean 3-space" (1927, [66]); "Note on projective coordinates" (1928, [67]); "On a problem in the theory of groups arising in the foundations of differential geometry" (1929, with H. Weyl, [68]); "Hypertensors" (1930, [69]); and "Groups of motions in spaces admitting absolute parallelism" (1932, [70]). Today, according to Google Scholar, his most cited paper is "The uncertainty principle" [71].

His association with Weyl went much further than the single paper mentioned above. In 1931 he published an English translation of the second edition of Weyl's classic text "The theory of groups and quantum mechanics" [72].

After he returned to Princeton, he was approached by the California Institute of Technology with the offer of a professorship. Princeton tried to counter the offer with inducements for him to remain there, but Robertson had been very happy at Caltech before. He accepted the position at Caltech and held it from 1947 until he died in 1961.

Applying his mathematical skills to cosmology had been an interest of Robertson's from early in his career, as the paper "On relativistic cosmology" (1928, [73]) shows.

The paper "Relativistic cosmology" from 1933, reprinted here [74], summarised his own and others' contributions to the subject up to that time. In three papers entitled "Kinematics and world structure", published in 1935 and 1936 [75-77], Robertson looked at Milne's theory and Einstein's theory of gravitation and their respective applications to cosmology, arguing strongly that Milne's objections to Einstein's theory are not valid. After moving from Princeton to Caltech, Robertson's interests became somewhat more directed towards astronomy. However, he continued to develop earlier themes with papers such as "On the present state of relativistic cosmology" (1949, [78]). A summary of the effects of general relativity and curved space in 1949 ("Geometry as a Branch of Physics", [79]) used only school mathematics.

The Poynting-Robertson effect is so named for Robertson's paper "Dynamical effects of radiation in the solar system" of 1937 [80], in which he studied the behaviour of a moving body subject to the laws of general relativity, which is absorbing and 
re-emitting solar radiation. A small dust particle in orbit around the Sun is constantly scattering sunlight. More photons are sent in the direction of travel than against it. In consequence, the particle suffers a backward push. This takes angular momentum away from the particle; therefore, the particle spirals inward toward the sun.

He was elected a member of the National Academy of Sciences of the United States in 1951 (and served as its foreign secretary from 1958 until his death), and of the American Academy of Arts and Sciences. His service to the Academy is memorialized by the H. P. Robertson Memorial Fund, established in 1962 by a group of personal friends and companies he advised. The fund is used for a lecture on any topic, at the Academy meeting, every third year. The first Robertson lecture was given by John Wheeler of Princeton, on relativity and geometry.

Robertson died on 26 August 1961 of a pulmonary embolism following an injury in a minor car accident.

Taub wrote in Ref. [63]: "Bob will be remembered by many for being a vital person with a quick wit, and for having an enormous appreciation for stories and limericks, some of which he originated. ... I warrant that many acknowledged and many unacknowledged footnotes are due to Robertson as a result of conversations originating around Angela's kitchen table."

Robertson's last contribution to science literature came 7 years after his death. One of his last students, Thomas W. Noonan, prepared his elegantly written notes as a book, Relativity and Cosmology, published in 1968 [81].

Several of Robertson's results remained unpublished. Among these, Noonan listed rigid body motion in special relativity, a study of Gödel's model, orbits around a variable mass, oscillation through the Schwarzschild singularity, and second-order plane gravitational waves.

More extended accounts of Robertson's biography can be found in Refs. [62], [63] and [82] below. In particular, Ref. [62] contains a complete list of Robertson's publications and a rather detailed account of his war-related activities.

\section{References}

1. Alpher, R.A., Bethe, H.A., Gamow, G.: The origin of chemical elements. Phys. Rev. 73, 803-804 (1948)

2. Barbour, J., Pfister, H. (eds.): Mach's Principle: From Newton's Bucket to Quantum Gravity, Einstein Studies, vol. 6. Birkhäuser, Boston (1995)

3. Bardeen, J.M.: Gauge-invariant cosmological perturbations. Phys. Rev. D 22, 1882-1905 (1980)

4. Bartelmann, M., Schneider, P.: Weak gravitational lensing. Phys. Rep. 340, 291-472 (2001)

5. Berger, A. (ed.) The Big bang and Georges Lematre: proceedings of a symposium in honour of G. Lematre fifty years after his initiation of big-bang cosmology, Louvain-la-Neuve, Belgium, 10-13 October 1983 (D. Reidel Pub. Co.) (1984)

6. Bondi, H.: Spherically symmetric models in general relativity. Mon. Not. R. Astron. Soc. 107, 410 (1948). Reprinted as a Golden Oldie: Gen. Relativ. Gravit. 31, 1777 (1999)

7. Bondi, H.: Cosmology. Cambridge University Press, Cambridge (1960)

8. Célérier, M.-N.: Do we really see a cosmological constant in the supernovae data? Astron. Astrophys. 353, 63-71 (2000) [arXiv:astro-ph/9907206]

9. Célérier, M.-N., Bolejko, K., Krasiński, A.: A (giant) void is not mandatory to explain away dark energy with a Lemaître-Tolman model. Astron. Astrophys. 518, A21 (2010)

10. Ehlers, J.: Beiträge zur relativistischen Mechanik kontinuerlicher Medien. Akad. Wiss. Lit. Mainz, Abhandl. Math.-Nat. K1. 11, 793 (1961). English translation: (1993), Gen. Relativ. Gravit. 25, 12251266 
11. Ehlers, J., Rindler, W.: A phase-space representation of Friedmann-Lemaître universes containing both dust and radiation and the inevitability of a big bang. Mon. Not. R. Astron. Soc. 238, 503-521 (1989)

12. Einstein, A.: Kosmologische Betrachtungen zur allgemeinen Relativitätstheorie. Sitzb. Preuss. Akad. Wiss: 142 (1917). English translation in The Principle of Relativity by Lorentz, H. A., Einstein, A., Minkowski, H., Weyl, H. (Dover: New York, 1923).

13. Ellis, G.F.R.: Relativistic Cosmology. In: Sachs, R.K. (ed.) General Relativity and Cosmology, Proc. Int. School of Physics "Enrico Fermi” (Varenna), Course XLVII, pp. 104-179. Academic Press, New York (1971). Reprinted as a Golden Oldie: Gen. Relativ. Gravit. 41, 581 (2009)

14. Ellis, G.F.R.: Topology and cosmology. Gen. Relativ. Gravit. 2, 7-21 (1971)

15. Ellis, G.F.R.: A History of cosmology 1917-1955. In: Howard, D., Stachel, J. (eds.) Einstein and the History of General Relativity. Einstein Study Series, Vol. 1, pp. 367-431. Birkhäuser, Boston (1990)

16. Ellis, G.F.R: Innovation resistance and change: the transition to the expanding universe. In: Bertotti, B., Balbinot, R., Bergia, S., Messina, A. (eds.) Modern Cosmology in Retrospect, pp. 97-114. Cambridge University Press, Cambridge (1990)

17. Ellis, G.F.R. (2006). Issue in the Philosophy of cosmology. In: Butterfield, J., Earman, J. (eds.) Handbook in Philosophy of Physics, pp. 1183-1285. Elsevier, Amsterdam (2006) [arxiv:astro-ph/0602280]

18. Ellis, G.F.R., Bruni, M.: A covariant and gauge-free approach to density fluctuations in cosmology. Phys. Rev. D 40, 1804-1818 (1989)

19. Ellis, G.F.R., Maartens, R.: The emergent universe: inflationary cosmology with no singularity. Class. Quant. Gravit. 21, 223-232 (2004) [gr-qc/0211082]

20. Ellis, G.F.R., Maartens, R., MacCallum, M.A.H.: Relativistic Cosmology. Cambridge University Press, Cambridge (2012)

21. Ellis, G.F.R., MacCallum, M.A.H.: A class of homogeneous cosmological models. Commun. Math. Phys. 12, 108-141 (1969)

22. Ellis, G.F.R., Murugan J., Tsagas, C.G.: The emergent universe: an explicit construction. Class. Quant. Gravit. 21, 233-249 [gr-qc/0307112] (2004)

23. Ellis, G.F.R., van Elst, H: Cosmological models. In: Lachièze-Ray, M. (ed.) Theoretical and Observational Cosmology (Cargese Lectures 1998), volume 541 of Nato Series C: Mathematical and Physical Sciences. Kluwer, Dordrecht, 1 (1999) [arXiv:gr-qc/9812046]

24. Etherington, I.M.H.: On the Definition of Distance in General Relativity. Philos. Mag., series 7 15, 761 (1933). Reprinted as a Golden Oldie: Gen. Relativ. Gravit. 39(7), 1055 (2007)

25. Friedmann, A.A.: Über die Krümmung des Raumes. Z. Physik 10, 377 (1922)

26. Friedmann, A.A.: Uber die Möglichkeit einer Welt mit konstanter negativer Krümmung des Raumes. Z. Physik 21, 326 (1924). Both papers by Friedmann reprinted as a single segment of the Golden Oldies. Gen. Relativ. Gravit. 31, 1985 (1999) + addendum: Gen. Relativ. Gravit. 32, 1937 (2000)

27. Gamow, G.: Expanding universe and the origin of the elements. Phys. Rev. 70, 572-573 (1946)

28. Gödel, K.: An example of a new type of cosmological solutions of Einstein's field equations of gravitation. Rev. Mod. Phys. 21, 447 (1949)

29. Gödel, K: Rotating universes in general relativity theory. pp. 175-181. In: Proceedings of the International Congress of Mathematicians in Cambridge, Massachusetts, U.S.A., August 30-September 6, 1950, I, (American Mathematical Society, Providence, 1952). Both papers reprinted as Golden Oldies in Gen. Relativ. Gravit. 32, 1399-1427 (2000)

30. Gregory, S.A., Thompson, L.A.: The Coma/A1367 supercluster and its environs. Astrophys. J. 222, 784 (1978)

31. Grishchuk, L.P.: Cosmological models and spatial homogeneity criteria. Astron. Zh. 44, 1097 (1967); English translation: Sov. Astron. A. J. 11, 881 (1968)

32. Joeveer, M., Einasto, J., Tago, E.: Spatial distribution of galaxies and of clusters of galaxies in the southern galactic hemisphere. Mon. Not. R. Astron. Soc. 185, 357-370 (1978)

33. Kirshner, R.P., Oemler, A.J., Schechter, P.L., Shectman, S.A.: A million cubic megaparsec void in Bootes. Astrophys. J. 248, L57 (1981)

34. Krasiński, A.: Inhomogeneous Cosmological Models. Cambridge University Press, Cambridge (1997)

35. Lachieze-Rey, M., Luminet J.-P.: Cosmic Topology. Phys. Rep. 254, 135-214 (1995) [arXiv:grqc/9605010]

36. Lemaître, G.: Un univers homogène de masse constante et de rayon croissant, rendant compte de la vitesse radiale de nébuleuses extra-galactiques. Ann. Soc. Sci. Bruxelles A 47, 49 (1927). English translation, partly re-edited: Mon. Not. Roy. Astron. Soc. 91, 483 (1931)

37. Lemaître, G.: L’Univers en expansion. Ann. Soc. Sci. Bruxelles A 53, 51 (1933). Reprinted as a Golden Oldie: Gen. Relativ. Gravit. 29, 637 (1997) 
38. Lifshitz, E.M.: On the gravitational stability of the expanding universe. J. Phys. USSR 10, 116 (1946) (Sov. Phys. JETP 16, 587)

39. Livio, M.: Lost in translation: mystery of the missing text solved. Nature 479, 171 (2011)

40. Luminet, J.-P.: Geometry and Topology in Relativistic Cosmology. In: Bartocci, C., Boi, L., Sinigaglia, C. (eds.) New Trends in Geometry, and Its Role in the Natural and Life Sciences. Elsevier, Amsterdam (2007) [arXiv:0704.3374]

41. Maartens, R.: Is the Universe homogeneous? Philos. Trans. R. Soc. A 369, 5115-5137 (2011) [arXiv:1104.1300]

42. Mukhanov, V.F., Feldman, H.A., Brandenberger, R.H.: Theory of cosmological perturbations. Phys. Rep. 215, 203-333 (1992)

43. Plebański, J., Krasiński, A.: An Introduction to General Relativity and Cosmology. Cambridge University Press, Cambridge (2006)

44. Peters, P., Uzan, J.-P.: Primordial Cosmology. Oxford University Press, Oxford (2009)

45. Rindler, W. (1956) Visual horizons in world models. Mon. Not. R. Astron. Soc 116, 662. Reprinted as a Golden Oldie: Gen. Relativ. Gravit. 34, 131 (2002)

46. Rindler, W.: Relativity: Special, General, and Cosmological. Oxford University Press, Oxford (2001)

47. Robertson, H.P.: On the foundations of relativistic cosmology. Proc. Nat. Acad. Sci. 15, 822-829 (1929)

48. Robertson, H.P.: Relativistic cosmology. Rev. Mod. Phys. 5, 62-90 (1933)

49. Robertson, H.P.: Kinematics and world-structure. Astrophys. J. 82, 284-301 (1935)

50. Schrödinger, E.: Expanding Universes. Cambridge University Press, Cambridge (1957)

51. Sandage, A.R.: The ability of the 200-inch telescope to discriminate between selected world models. Astrophys. J. 133, 355 (1961)

52. Schneider, P., Ehlers, J., Falco, E.E.: Gravitational Lenses. Springer, Berlin (1999)

53. Sen, N.R.: On the stability of cosmological models. Z. Astrophysik 9, 215 (1934). Reprinted as a Golden Oldie Gen. Relativ. Gravit. 29, 1473 (1997)

54. Szekeres, P.: A class of inhomogeneous cosmological models. Commun. Math. Phys. 41, 55 (1975)

55. Szekeres, P.: Quasispherical gravitational collapse. Phys. Rev. D 12, 2941 (1975)

56. Tolman, R.C.: Effect of inhomogeneity on cosmological models. Proc. Nat. Acad. Sci. USA 20, 169 (1934). Reprinted as a Golden Oldie: Gen. Relativ. Gravit. 29, 931 (1997)

57. Walker, A.G.: On Riemannian spaces with spherical symmetry about a line, and the conditions for isotropy in general relativity. Q. J. Math. Oxf. Ser. 6, 81 (1935)

58. Walker, A.G.: On Milne's theory of world-structure. Proc. Lond. Math. Soc. 42, 90 (1937)

59. Walker, A.G.: Completely symmetric spaces. J. Lond. Math. Soc. 19, 216-226 (1944)

60. Weyl, H.: Zur allgemeinen Relativitätstheorie. Physikalische Zeitschrift 24, 230-232 (1923). Reprinted as a Golden Oldie Gen. Relativ. Gravit. 41(7), 1655 (2009)

61. O’Connor, J.J., Robertson, E.F.: http://www-history.mcs.st-and.ac.uk/Biographies/Robertson.html

62. Greenstein, J.L.: Howard Percy Robertson January 27, 1903-August 26, 1961. Biographical Memoirs National Academy of Sciences 51, 343-361 (1980). Also available from http://books.nap.edu/html/ biomems/hrobertson.pdf

63. Taub, A.H.: H.P. Robertson: 1903-1961. In: H.P. Robertson: January 27, 1903-August 26, 1961. In memoriam. Society for Industrial and Applied Mathematics, Philadelphia, Pa., pp. 3-10 (1963)

64. Robertson, H.P.: Bull. Am. Math. Soc. 30, 14 (1924)

65. Robertson, H.P.: Proc. Natl. Acad. Sci. USA 11, 590-592 (1925)

66. Robertson, H.P.: Trans. Am. Math. Soc. 29, 481-496 (1927)

67. Robertson, H.P.: Proc. Natl. Acad. Sci. USA 14, 153-154 (1928)

68. Robertson, H.P., Weyl, H.: Bull. Am. Math. Soc. 35, 686-690 (1929)

69. Robertson, H.P.: Ann. Math. 31, 281-291 (1930)

70. Robertson, H.P.: Ann. Math. 33, 496-520 (1932)

71. Robertson, H.P.: Phys. Rev. 34, 163-164 (1929)

72. Weyl, H.: Theory of Groups and Quantum Mechanics. E. P. Dutton, New York (1931)

73. Robertson, H.P.: Philos. Mag. 5, 835-848 (1928)

74. Robertson, H.P.: Rev. Mod. Phys. 5, 62-90 (1933)

75. Robertson, H.P.: Astrophys. J. 82, 284-301 (1935)

76. Robertson, H.P.: Astrophys. J. 83, 187-201 (1936)

77. Robertson, H.P.: Astrophys. J. 83, 257-271 (1936)

78. Robertson, H.P.: Proc. Am. Philos. Soc. 93, 527-531 (1949)

79. Robertson, H.P.: In: Schilpp, P.A. (ed.) Albert Einstein: Philosopher Scientist, pp. 315-332. Tudor Publishing, New York (1949) 
80. Robertson, H.P.: Mon. Not. R. Astron. Soc. 97, 423-438 (1937)

81. Robertson, H.P., Noonan, T.W.: Relativity and Cosmology. W.B. Saunders Company, Philadelphia (1968)

82. Robertson, H.P.: January 27, 1903-August 26, 1961. In memoriam. J. Soc. Ind. Appl. Math. 10(4), 737-801 (1962) 